\title{
Large Coherence Area Thin-Film Photonic Stop-Band Lasers
}

\author{
Victor I. Kopp and Azriel Z. Genack \\ Department of Physics, Queens College of CUNY, Flushing, New York 11367 \\ and Chiral Photonics, Inc., Clifton, New Jersey 07012 \\ Zhao-Qing Zhang \\ Physics Department, Hong Kong University of Science and Technology, Clear Water Bay, Kowloon, Hong Kong, China
}

(Received 5 October 2000)

\begin{abstract}
We demonstrate that the shift of the stop-band position with increasing oblique angle in periodic structures results in a wide transverse exponential field distribution corresponding to strong angular confinement of the radiation. The beam expansion follows an effective diffusive equation depending only upon the spectral mode width. In the presence of gain, the beam cross section is limited only by the size of the gain area. As an example of an active periodic photonic medium, we calculate and measure laser emission from a dye-doped cholesteric liquid crystal film.
\end{abstract}

DOI: 10.1103/PhysRevLett.86.1753

PACS numbers: 42.70.Qs, 42.70.Df

Photonic band-gap materials hold promise as a platform for a new generation of efficient, compact photonic devices. The initial excitement generated by these materials has been sustained by the discovery of new physical effects leading to new applications [1-3]. Yablonovitch has predicted that the lasing threshold can be reduced by introducing a defect into an otherwise periodic photonic band-gap structure. Since spontaneous emission is suppressed in the band gap, excitation will not then be drained by modes other than the lasing mode [2]. In addition, the long dwell time of such localized defect modes reduces the gain required to reach the lasing threshold. Lasing has recently been observed in 2D photonic crystals [4], and promising 3D photonic crystals have been fabricated [5]. The threshold for lasing may even be suppressed at defect modes in periodic structures that do not possess a full 3D photonic band gap, such as in 1D periodic samples, including vertical cavity surface emitting lasers (VCSELs) [6]. Recently, lasing at the band edge has been demonstrated in dye-doped cholesteric liquid crystals (CLCs). In these chiral structures, a stop band exists for circularly polarized light that has the same sign of rotation as the CLC structure. Since the dwell time within the sample for emitted photons is enhanced near the band edge, the lasing threshold is also substantially reduced [7].

In this Letter, we present an unexpected conjunction of strong expansion of the region of phase coherence within the medium and weak divergence of the output beam, which is made possible in a periodic structure possessing a photonic stop band. Our calculations show that a diffusionlike spreading of the coherent field leads to wide area lasing at the edge of a photonic stop band or at a localized state in the middle of the stop band. These results are supported by measurements of the spatial profile of laser emission from a promising organic material - a dye-doped cholesteric liquid crystal. Since the maximum excitation energy is proportional to the laser area, large-area thin-film devices provide a new approach for high-power lasers. While liquid crystals are not stable at the elevated temperatures produced by intense laser excitation, polymeric CLCs may be promising materials for large-area high-power lasers.

Though lasing has not yet been observed in 3D periodic structures, the enhanced photon dwell times and microcavity effects in localized states have suppressed the laser threshold in lower dimensional periodic systems. Unlike lasers based on Fabry-Perot resonators, the band-edge and defect modes of 1D structures are significantly separated from adjacent modes and have narrower linewidth than other modes. They may therefore operate as single mode lasers over a significant power range. The shift of the band gap with oblique angle at the band-edge frequency permits only the propagation of light with wave-vector components near the normal. Unlike conventional spreading of light between two mirrors, which requires oblique wave-vector components [Fig. 1(a)], photonic band structures lead to enhanced spreading of light with only near-normal wavevector components [Fig. 1(b)].

It would appear that VCSELs are the best candidate for large area lasing in a 1D periodic structure. But high order transverse modes arise [8] in small-diameter VCSEL, while in large-diameter VCSELs spontaneous filamentation results from structural nonuniformities [9]. Other 1D structures that can be produced as a macroscopic single domain material are self-organized cholesteric liquid crystals [10]. Lasing at the edge of the stop band of CLCs has recently been demonstrated [7], and here we will specifically consider measurements and simulations of the spatial distribution of light in these materials.

The molecular director in cholesteric structures lies in a molecular plane and executes a helical rotation perpendicular to the planes. A stop band centered at a wavelength in the medium equal to the helical pitch $P$ is created for waves propagating along the helical axis. Optically pumped lasing in dye-doped CLCs occurs at the first mode at either the high or low frequency edge of the stop band, depending 

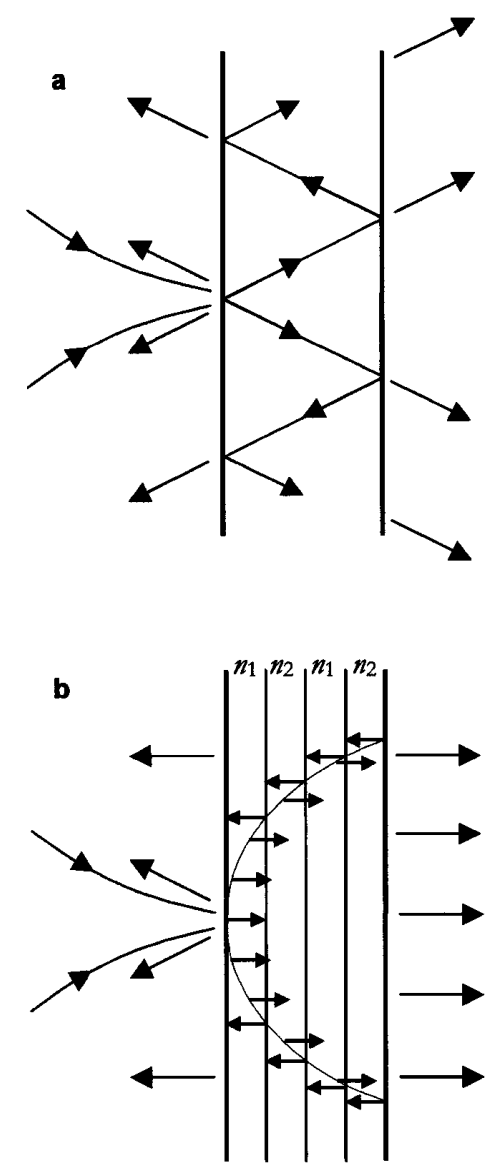

FIG. 1. Coherent spreading of beam inside an amplifying medium. (a) Two flat mirrors define a cavity, in which spreading of the beam involves oblique wave vector components. The resulting beam size is linearly proportional to the photon dwell time. (b) Layered photonic band-gap medium, in which oblique components of the wave vector are reflected, except for wave-vector components close to normal. The beamwidth follows a diffusion equation and is proportional to the square root of the photon dwell time.

on the position of the dye emission maximum. These occur at vacuum wavelengths of $n_{e} P$ or $n_{o} P$, where $n_{e}$ and $n_{o}$ are the extraordinary and ordinary indices of refraction, respectively. For the same gain, slightly above threshold, lasing generally occurs only in the narrowest mode, which is the first mode on the high frequency side of the band. This mode is composed of two circularly polarized counterpropagating components traveling normal to the layers of the structure resulting in an electric field, which is everywhere aligned with the lowest index axis of the optical indicatrix within the molecular layer plane. For off-normal incident radiation, the gap shifts to higher frequency, placing oblique waves at the frequency of the lasing mode inside the stop band. As a result, transmitted or emitted radiation is confined to a narrow range of angles about the normal direction as shown in Fig. 1(b). The restricted angular distribution corresponds to a wide beam at the output surface of the film.
The CLC films studied possessed a right-handed structure with $n \sim 1.7$ and $\Delta n \sim 0.2$ and a thickness of $35 \mu \mathrm{m}$. The samples were doped with laser dye PM-597 with an absorption peak at $530 \mathrm{~nm}$ and an emission peak at $590 \mathrm{~nm}$ and pumped with $150 \mathrm{~ns}$ pulses of frequency doubled $Q$-switched Nd:YAG laser at $532 \mathrm{~nm}$. The sample lases at the mode closest to the upper band edge. The emitted beam is perpendicular to the CLC film. The intensity profile on a screen at a distance of $11.5 \mathrm{~cm}$ from the sample is shown in the inset of Fig. 3 (below).

Simulations of propagation in a CLC medium are carried out in model media of successive anisotropic amplifying layers with thickness $h$ significantly smaller than the wavelength of the incident light. The direction of the molecular axis is rotated between layers by an angle $2 \pi h / P$. A normally incident circularly polarized 1D Gaussian beam with the helicity of the CLC structure and frequency of the lasing mode is incident upon the sample. For simplicity of calculation, we consider an incident beam, which is a superposition of waves with an intensity distribution that is Gaussian in the plane of incidence (the $x-z$ plane) and homogeneous in the perpendicular direction. The main results obtained for this wave are readily generalized to the case of a Gaussian beam. Transmission is calculated using a $4 \times 4$ transfer matrix method [11-13] for each Fourier component of the beam. These are superposed to give the transmitted wave.

The transfer matrix is computed numerically for each plane wave component by taking the product of the transfer matrix for each layer of thickness $h$ with uniform dielectric tensor. The value of $h$ is small enough that the value of the transmittance does not change significantly if it is decreased further. Transmission is simulated in a CLC film with $P=370 \mathrm{~nm}$, thickness $L=35 \mu \mathrm{m}$, and extraordinary and ordinary dielectric constants $\varepsilon_{e}$ and $\varepsilon_{o}$ which correspond to refractive indices $n_{e}=1.8$ and $n_{o}=1.6$. For the background medium, we use $n_{b}=1.52$, the refractive index of the glass substrates. Gain is introduced by adding a negative imaginary part to both $\varepsilon_{e}$ and $\varepsilon_{o}$. The width of the band-edge mode in the transmission spectrum drops with increasing gain and vanishes at a critical value of the gain corresponding to the laser threshold. At this point, both the transmittance and reflectance diverge.

The simulations show that the wave function at the output surface for a gain coefficient slightly below the critical value is proportional to $\exp [-\alpha(1-i)|x|]$ at the frequency of the band-edge state for the perpendicularly propagating wave. A typical intensity distribution is shown in linear and logarithmic plots in Fig. 2. The intensity distribution is seen to decay exponentially, $I \propto \exp (-2 \alpha|x|)$. Except for a small region near the beam center, the phase of the electric field increases linearly with the same coefficient $\alpha$, i.e., $\phi=\alpha|x|$. The value of $\alpha$ decreases with increasing gain coefficient and vanishes at the critical gain. The value of $\alpha$, and, hence, the wave function, is found to be independent of the width of the incident beam. 


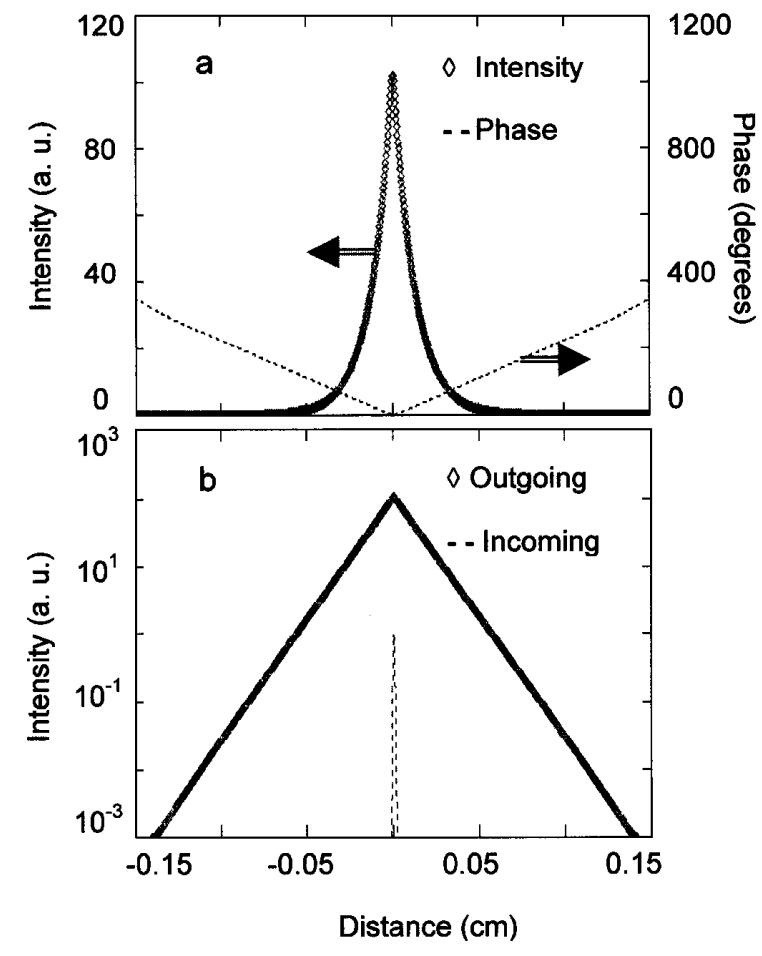

FIG. 2. Computer simulation of spatial distribution of laser emission at output surface of CLC. (a) Intensity and phase in linear scale; (b) intensities of incoming and outgoing beams in semilog scale.

The calculated intensity distribution at the output surface gives rise to an oscillatory structure in the far field. The measured and calculated far-field intensities are shown in Fig. 3 at a distance of $11.5 \mathrm{~cm}$ from the sample. The agreement is good outside of the central region, but calculations give a flatter peak than is measured. This is due to the use of a beam with a Gaussian intensity distribution only in one direction in the calculation and to the finite gain region created by the pump laser beam.

The spatial distribution can be shown analytically to be a consequence of the Lorentzian shape of the single resonant peak of the transmitted electric field,

$$
T_{\beta}(k) \approx \frac{C_{\beta}}{B-i\left(k-k_{r}\right)} ; \quad \beta=x, y, z,
$$

where $C_{\beta}$ and $B$ are constants, $k=2 \pi n_{b} / \lambda$ and $k_{r}=$ $2 \pi n_{b} / \lambda_{r}$ are wave vectors in the embedding and resonant medium, respectively. The linewidth of the transmission spectrum is $\Delta \lambda=B \lambda_{r}^{2} / 2 \pi n_{b}$. When the gain coefficient $\gamma$ is slightly below its critical value $\gamma_{c}, B$ is proportional to $\left(\gamma_{c}-\gamma\right)$. The wave incident at the oblique angle $A$ decouples into a product of waves propagating in the transverse and $z$ directions, making it possible to generalize Eq. (1) by replacing $k$ by $k \cos A$. In the limit of $B \ll k_{r}$ at the band-edge frequency $k=k_{r}$, the transmittance is strongly peaked in the normal direction, and we can expand $\cos A \cong 1-A^{2} / 2$. Equation (1) then gives

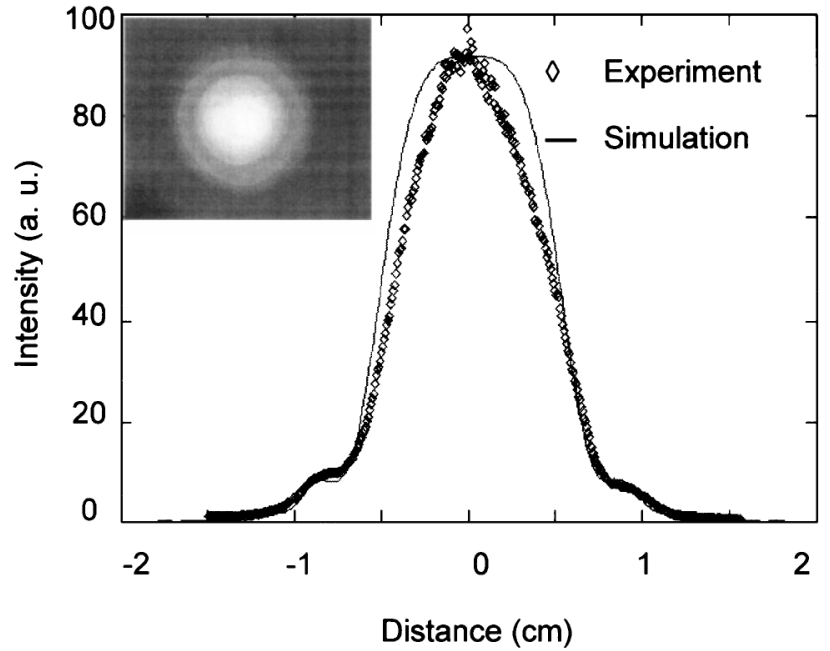

FIG. 3. Spatial distribution of laser emission in far field (11.5 $\mathrm{cm}$ from sample). The charge-coupled device image of the intensity profile is shown in the inset.

$$
T_{\beta}\left(A, k=k_{r}\right) \approx \frac{2 C_{\beta}}{2 B+i A^{2} k_{r}} .
$$

Using Eq. (2) and superposing all transmitted plane waves for the incident Gaussian beam, we obtain the transmitted electric field,

$$
E_{\beta}^{T}(x, z=L) \propto \exp \left[-(1-i) \sqrt{B k_{r}}|x|\right] .
$$

This is precisely the wave function indicated in Fig. 2 with $\alpha=\sqrt{B k_{r}}$. The wave function obtained in Eq. (3) depends only on $B k_{r}$ and is independent of the spatial extent of the incident beam. Since $B$ is proportional to $\left(\gamma_{c}-\gamma\right)$, the wave function becomes an unbounded plane wave as the gain approaches the critical value. When $|x| \ll 1 / \sqrt{B k_{r}}$, Eq. (3) becomes invalid, and it cannot describe the central region of the beam.

The width of the beam is defined as $W \equiv 2 x_{0}$, where $x_{0}$ is the position at which the intensity drops to half its peak value. Using Eq. (3), this gives a beamwidth at the output surface of $W=\ln 2 / \sqrt{B k_{r}}$. By substituting $2 \Delta \lambda=$ $B \lambda_{r}^{2} / \pi n_{b}$ and $k_{r}=2 \pi n_{b} / \lambda_{r}$ into the previous relation, we find a universal relation between $W$ and the linewidth $2 \Delta \lambda$ in transmission for normally incident radiation at $\lambda_{r}$,

$$
\frac{\lambda_{r}}{n_{b} W}=\frac{\sqrt{2} \pi}{\ln 2} \sqrt{\frac{2 \Delta \lambda}{\lambda_{r}}} .
$$

This relation is valid as long as $B \ll k_{r}$, which holds if the transmission linewidth is sufficiently narrow, as in thick or highly reflective samples, or if the gain is close to the laser threshold. By the condition that the medium has a single transmission peak, we indicate that the angular separation between modes at a given frequency exceeds the angular spread in the incident beam. This can be achieved at the first mode at the high frequency band edge or at a localized mode within the band gap for samples possessing sufficiently large index contrast. It may also be achieved in 


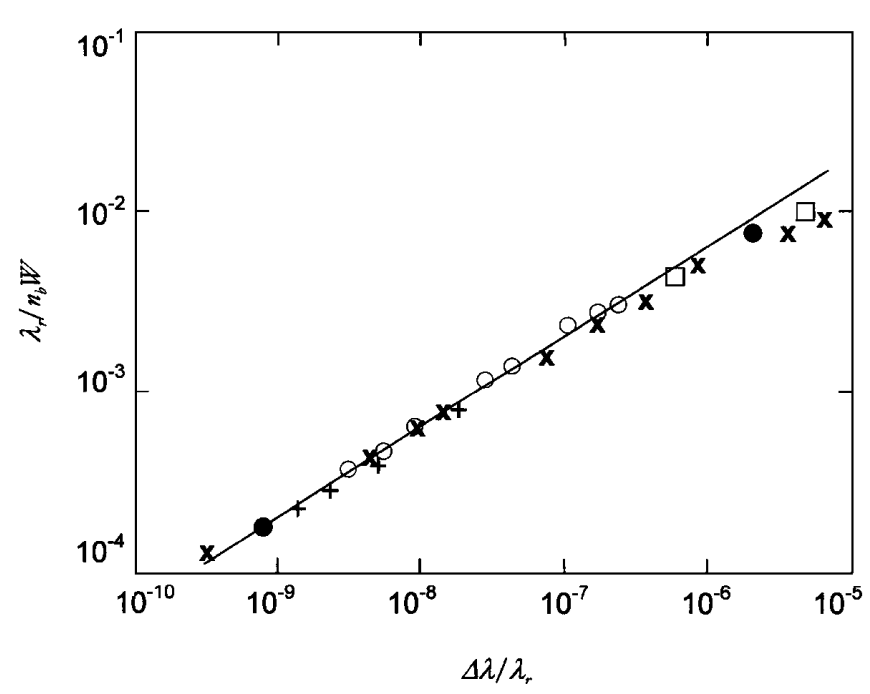

FIG. 4. Comparison of the universal relation for the dependence of the inverse beamwidth upon relative linewidth of Eq. (4), shown as a solid line, with simulations of transmission at the band edge of CLC $(O)$ and BL $(+)$ systems and in FP $(\times)$ systems with different values of gain, through CLC samples of different thickness $(\square)$, and at localized states in the middle of the band gap of BL ( samples of different thickness.

a microcavity Fabry-Perot (FP) interferometer with mirror separation of the order of the wavelength. This is confirmed in simulations, shown in Fig. 4, for transmission at the band-edge mode in CLC $(\mathrm{O})$, and binary layered $(\mathrm{BL})(+)$ systems and in thin FP $(\times)$ systems with different values of gain. Simulations are also carried out for two CLC samples without gain but with different thickness $(\square)$ and for a localized mode in BL (O) samples containing a single defect in the middle of the sample. Good agreement is found between the results of simulations and Eq. (4), which is shown as the solid line.

The universal relation in Eq. (4) has a surprising interpretation in terms of diffusion. Since the photon dwell time $\tau \approx \lambda_{r}^{2} /(2 \pi c \Delta \lambda)$ is inversely proportional to the transmission linewidth, Eq. (4) can be interpreted in terms of the diffusion relation: $W^{2} \approx D \tau$ with an effective diffusion constant $D \approx c \lambda_{r} / 2 \pi n_{b}^{2}=c / n_{b} k_{r}$. This effective diffusion constant is proportional to the wavelength of the resonant mode and is independent of the sample thickness and gain coefficient. Although we have assumed the wave is homogeneous in the $y$ direction, this condition can be relaxed in the analytical approach. If the incident beam is a Gaussian wave in both the $x$ and $y$ directions, the generalization of the $1 \mathrm{D}$ approach leads to an outgoing wave: $E_{\beta}^{T} \propto(1 / \sqrt{\rho}) \exp \left[-(1-i) \sqrt{B k_{r}} \rho\right]$, where $\rho=\sqrt{x^{2}+y^{2}}$ as long as the condition $\rho \gg 1 / \sqrt{B k_{r}}$ is satisfied. This is similar to the wave function found in Eq. (3).

In conclusion, our calculations and measurements have shown that wide area lasing with beamwidths greatly exceeding the sample's thickness can be created in a thin film at the edge of a stop band or at a localized state in the middle of the gap. This is possible at the laser wavelength because only a single mode of radiation exists over a wide angular range centered at the normal direction. The spread of optical coherence is diffusionlike, resulting in a beamwidth, which is proportional to the square root of the photon dwell time. The use of large-area, thin-film lasers facilitates heat extraction and high-power operation. These may enable lightweight optical sources for free-space communication, coherent backlighting for 3D holographic and projection displays, and therapeutic irradiation of large areas of skin.

This work was supported by the National Science Foundation under Grant No. DMR9973959, and by the Army Research Office under Grant No. DAAD19-00-1-0362. It was performed in part under the auspices of the City University of New York CAT in Ultrafast Photonic Materials and Applications.

[1] J. D. Joannopoulos, R. D. Meade, and J. N. Winn, in Photonic Crystals: Molding the Flow of Light (Princeton University, Princeton, NJ, 1995).

[2] E. Yablonovitch, Phys. Rev. Lett. 58, 2059 (1987).

[3] S. John, Phys. Rev. Lett. 58, 2486 (1987).

[4] O. Painter, R. K. Lee, A. Scherer, A. Yariv, J. D. O'Brien, P. D. Dapkus, and I. Kim, Science 284, 1819 (1999).

[5] A. Blanco et al., Nature (London) 405, 437 (2000).

[6] H. Yokoyama and K. Ujihara, in Spontaneous Emission and Laser Oscillation in Microcavities (CRC Press, Boca Raton, Florida, 1995).

[7] V.I. Kopp, B. Fan, H. K. M. Vithana, and A.Z. Genack, Opt. Lett. 23, 1707 (1998).

[8] J.E. Epler, S. Gehrsitz, K.H. Gulden, M. Moser, H. C. Sigg, and H. W. Lehmann, Appl. Phys. Lett. 69, 722 (1996).

[9] M. Orenstein, E. Kapon, N. G. Stoffel, J. P. Harbison, L. T. Florez, and J. Wullert, Appl. Phys. Lett. 58, 804 (1991).

[10] S. Elston and R. Sambles, in The Optics of Thermotropic Liquid Crystals (Taylor \& Francis, Bristol, PA, 1998).

[11] S. Teitler and B. Henvis, J. Opt. Soc. Am. 60, 830 (1970).

[12] D. W. Berreman, J. Opt. Soc. Am. 62, 502 (1972).

[13] H. Wohler, G. Haas, M. Fritsch, and D. A. Mlynski, J. Opt. Soc. Am. A 5, 1554 (1988). 